\title{
V-set and transmembrane domain-containing 1 is silenced in human hematopoietic malignancy cell lines with promoter methylation and has inhibitory effects on cell growth
}

\author{
TING LI $^{1-3}$, XIAOHUAN GUO ${ }^{1-3}$, WENYAN WANG ${ }^{1-3}$, XIAONING MO $^{1-3}$, \\ PINGZHANG WANG ${ }^{1-3}$ and WENLING HAN ${ }^{1-3}$ \\ ${ }^{1}$ Peking University Center for Human Disease Genomics; ${ }^{2}$ Key Laboratory of Medical Immunology, \\ Ministry of Health; ${ }^{3}$ Department of Immunology, School of Basic Medical Sciences, \\ Peking University Health Science Center, Beijing 100191, P.R. China
}

Received December 7, 2013; Accepted September 24, 2014

DOI: $10.3892 / \mathrm{mmr} .2014 .2785$

\begin{abstract}
Numerous leukocyte differentiation antigens act as important markers for research, diagnosis, triage and eventually treatment targets for hematopoietic malignancies. V-set and transmembrane domain-containing 1 (VSTM1) was identified by immunogenomic analysis as a potential leukocyte differentiation antigen gene. VSTM1 is located at 19q13.4 on human chromosomes, an important genomic region prone to genetic and epigenetic modifications in numerous hematopoietic malignancies. VSTM1-v1, a primary splicing form encoded by VSTM1, is a type I transmembrane molecule with an extracellular immunoglobulin V-like domain and two cytoplasmic immunoreceptor tyrosine-based inhibitory motifs. In the present study, VSTM1 expression was examined in normal human peripheral leukocytes and hematopoietic tumor cell lines; in addition, the aberrant methylation of the VSTM1 gene was evaluated using methylation-specific polymerase chain reaction (MSP). The results of the present study demonstrated that VSTM1 was widely expressed in normal human peripheral blood leukocytes, including granulocytes and monocytes, in concurrence with previous studies, as well as lymphocytes; in addition, the molecular size and expression levels of VSTM1 varied considerably between leukocytes. However, VSTM1 was undetectable in numerous hematopoietic tumor cell lines following promoter hypermethylation. The effects of pharmacologically-induced demethylation of the VSTMI gene and promoter region were
\end{abstract}

Correspondence to: Professor Wenling Han, Department of Immunology, School of Basic Medical Sciences, Peking University Health Science Center, 38 Xueyuan Road, Beijing 100191, P.R. China

E-mail: hanwl@bjmu.edu.cn

Key words: v-set and transmembrane domain containing 1, hematopoietic malignancy, methylation, cell growth, leukocyte differentiation antigen analyzed using MSP and biosulfite genomic sequencing, and the results revealed that VSTM1 expression was restored in methylation-silenced Jurkat cells. In addition, CKK-8 assays revealed that VSTM1-v1 overexpression in Jurkat cells resulted in growth suppression. Furthermore, the inhibitory effect on cell growth was enhanced following antibody-induced cross-linking of VSTM1-v1. In conclusion, the results of the present study indicated that promoter methylation silenced VSTM1 and negatively regulated cell growth in human hematopoietic malignancy cell lines.

\section{Introduction}

Leukocyte differentiation antigens are a series of glycoproteins and glycolipids attached to or inserted into the membrane of leukocytes, which are the major cells of the immune system and malignant cells that derive from them (1). These antigens are able to transmit membrane signals, regulate cell activation and differentiation as well as mediate numerous interactions between the immune system and antigens, other physiological systems or other components of the immune system (1-3). Numerous leukocyte differentiation antigens were reported to be involved in the pathogenesis and development of hematopoietic malignancies; in addition, certain antigens may be used as markers for diagnosis, classification, risk stratification and eventually as treatment targets in these malignant diseases $(4,5)$.

In previous unpublished experiments conducted in 2008 , we aimed to identify novel potential leukocyte differentiation antigens using an integrated bioinformatics analysis at a genome-wide level to select proteins matching the following criteria: 1 , No reported functional association with the immune system; 2, predominant expression in cells of the immune system; 3, high expression in certain specific subsets of immune cells; and 4, type I and type II membrane proteins preferred. $\mathrm{V}$-set and transmembrane domain-containing 1 (VSTM1) was identified by the data mining as a candidate gene. VSTM1 was found to be located at human chromosome 19q13.4 and to encode two primary splicing forms, VSTM1-v1 and VSTM1-v2. VSTM1-v1 contains 236 amino acids and is a type I membrane molecule, which has an immunoglobulin 
(Ig)V-like domain in its extracellular region and two immunoreceptor tyrosine-based inhibitory motifs (ITIMs) in its cytoplasmic tail. It has been suggested that VSTM1-v1 may be a novel ITIM-bearing inhibitory immune receptor involved in the regulation of phagocytes (6). VSTM1-v2 is composed of 205 amino acids and was reported to be a classical secretory glycoprotein, which does not contain a transmembrane domain. It was also reported that recombinant VSTM1-v2 promoted the differentiation and activation of human Th17 cells (7).

Genetic instabilities are widely accepted to be one of the major causes of cancer pathogenesis in humans. Chromosome 19q13 has been reported to be involved in numerous types of cancer, including hematopoietic malignancies, due to its instable genomic region. These instabilities include chromosome translocations, single nucleotide polymorphisms, amplifications, mutations and deletions, which occur frequently in certain types of myeloid and lymphoid leukemia and lymphoma (8-12). Furthermore, epigenetic modifications such as DNA methylation were reported to be involed in the dysregulation of genes located on 19q13.3-4 (13-15).

DNA methylation is an epigenetic code that controls the expression of lineage- and development-specific genes. It has been reported that the aberrant methylation of genes regulating the differentiation of hematopoietic lineages induced leukemic transformations (16-19). The aim of the present study was to examine the expression of VSTM1 in normal human peripheral blood leukocytes and hematopoietic tumor cell lines.

\section{Materials and methods}

Isolation of peripheral blood granulocytes, monocytes and lymphocytes. Peripheral blood mononuclear cells (PBMCs) were isolated from granulocytes and red blood cells in the buffy coats of healthy donors [obtained at the Beijing Red Cross Blood Center (Beijing, China) between December 2012 and April 2013] using Ficoll/Hypaque density-gradient centrifugation, as described previously (20), and cultured in RPMI 1640 medium supplemented with $10 \%$ fetal calf serum (FCS; Hyclone Laboratories, Inc, Logan, UT, USA) in petri dishes to enable monocyte adhesion. Non-adherent cells, predominantly peripheral blood lymphocytes (PBLs), and adherent monocytes were collected separately (20). Granulocytes were isolated through lysing red blood cells with a hypotonic buffer as previously described (21). Protocols performed in the present study were approved by the Ethics Committee of Peking University Health Science Center (Bejing, China). Written informed consent was obtained from all healthy donors.

Cell lines. KG-1, HL-60, HuT-78, Jurkat, K562, MOLT-4, Raji, Thp-1, U937 and RPMI 8866 cells, purchased from the American Type Culture Collection (Manassas, VA, USA), were cultured in RPMI 1640 medium supplemented with $10 \% \mathrm{FCS}$ at $37^{\circ} \mathrm{C}$ in a humidified atmosphere with $5 \% \mathrm{CO}_{2}$. Jurkat cells were treated with $2 \mu \mathrm{mol} / 1$ 5-aza-2'-deoxycytidine (Sigma-Aldrich, St. Louis, MO, USA) for 3 days, and a proportion of these cells were then further treated with $100 \mathrm{nmol} / \mathrm{l}$ trichostatin A (Cell Signaling Technology, Inc., Danvers, MA, USA) for $16 \mathrm{~h}$ as previously described $(22,23)$. Genomic DNA samples were extracted from Jurkat cells using a ZR Genomic
DNA II Kit ${ }^{\mathrm{TM}}$ (Zymo Research. Irvine, CA, USA) according to the manufacturer's instructions. Jurkat cell transfection was performed using electroporation; in brief, $25 \mu \mathrm{g}$ plasmid was mixed with $2 \times 10^{7}$ cells in $350 \mu \mathrm{l}$ serum-free medium and electroporated at a single pulse $(135 \mathrm{~V} ; 20 \mathrm{~ms})$ in a $2-\mathrm{mm}$ gap cuvette using an ECM 830 Square Wave Electroporation System (BTX Harvard Apparatus, Holliston, MA, USA).

Semiquantitative reverse transcription polymerase chain reaction $(R T-P C R)$. Total RNA was extracted from the cells using TRIzol reagent (Life Technologies Inc., Carlsbad, CA, USA). Reverse transcription was performed according to standard protocols using the RevertAid ${ }^{\mathrm{TM}}$ II First Strand complementary (c)DNA synthesis kit (Fermentas, Waltham, MA, USA). Human Multiple Tissue and Immune System MTC $^{\mathrm{TM}}$ panels containing cDNA from a pool of donors were purchased from Clontech Laboratories, Inc. (Mountain View, CA, USA). Semiquantitative PCR was performed as previously described (7).GAPDH was then amplified and used as an internal control. qPCR was performed as previously described (23) using the primers 5'-ACTTGCAGCTGGTGGTCACA-3' and 5'-CCGGAAGTTTGGAATGGCT-3'. Samples with unspecific amplification according to the dissolving curve and electrophoresis were regarded as zero. All samples were normalized to GAPDH using the comparative $\mathrm{Ct}$ method $(\Delta \Delta \mathrm{Ct})$.

Cytosine-phosphate-guanine (CpG) island screening, DNA bisulfite treatment and methylation analysis. $\mathrm{CpG}$ island searcher (http://cpgislands.usc.edu/) was used to screen for $\mathrm{CpG}$ islands in the promoter region of VSTM1. Based on the search results, primers for the detection of methylated or unmethylated alleles of the VSTM1 promoter and for bisulfite genomic sequencing (BGS) were designed. Bisulfite modification of DNA, methylation-specific PCR (MSP) and BGS were then performed as previously described $(22,23)$. The bisulfite-treated DNA was amplified using the methylation-specific primer set m3, 5'-TTGTATATTTTGGGGACGAATC-3' and m4, 5'-AAAAAAAATTCTACGATCATAACG-3' or the unmethylation-specific primer set u3, 5'-TTTGTATAT TTTGGGGATGAATT-3' and u4, 5'-AAAAAAAAA TTCTACAATCATAACA-3' (Beijing AuGCT DNA-SYN Biotechnology Co Ltd., Beijing, China). MSP was performed for 40 cycles using Ampli-Taq Gold (Perkin Elmer Applied Systems, Foster City, CA, USA) and hot start. MSP primers were tested in order to confirm that they did not amplify unbisulfited DNA; in addition, the specificity of MSP was confirmed by direct sequencing of PCR products. For BGS, bisulfite-treated DNA was amplified using the following primers: b1, 5'-AGAGTGGGGTAGAGAATTAGAGTGTTA -3 ' and b2, 5'-ACCTAAACTACCACTCCCACATAAAT-3'. The PCR products were cloned into the pGEM-T easy vector (Promega Corp., Maddison, WI, USA) and sequenced using ten randomly selected colonies.

Flow cytometric analysis. Cells were blocked using fluorescence-activated cell sorting (FACS) buffer [phosphate-buffered saline (PBS) containing 2\% FCS] on ice for $30 \mathrm{~min}$. A total of $1 \times 10^{6}$ cells were then incubated on ice for $1 \mathrm{~h}$ with rabbit anti-VSTM1 polyclonal antibody (prepared by authors as previously described; $2 \mu \mathrm{g} / \mathrm{sample}$ in $100 \mu \mathrm{l}$ FACS buffer) (24). 
Cells were then washed in PBS and stained with fluorescein isothiocyanate-conjugated anti-rabbit immunoglobulin (Ig; dilution, 1:100 in FACS buffer; Biolegend, Inc., San Diego, CA, USA) in the dark at $4^{\circ} \mathrm{C}$ for $30 \mathrm{~min}$. Cells were susequently washed in PBS and analyzed using a BD FACSCalibur flow cytometer (Becton-Dickinson, Franklin Lakes, NJ, USA).

Protein extraction and western blot analysis. Cells were harvested and lysed using $20 \mathrm{mmol} / 1$ Tris- $\mathrm{HCl}(\mathrm{pH} \mathrm{7.5)}$, $150 \mathrm{mmol} / 1 \mathrm{NaCl}, 1 \mathrm{mmol} / 1$ EDTA, $1 \%$ Triton X-100, and $1 \%$ protease inhibitor cocktail (Pierce, Rockford, IL, USA). Proteins were applied to $10 \%$ SDS-PAGE and electrotransferred onto polyvinylidene difluoride membranes (Hybond ${ }^{\circledR}$ Inc., Escondido, CA, USA). VSTM1 was detected using rabbit anti-VSTM1 polyclonal antibodies (pAb; $2 \mu \mathrm{g} / \mathrm{ml}$ ) and secondary horseradish peroxidase-conjugated goat anti-rabbit IgG antibodies (dilution, 1:5,000; Cell Signaling Technology, Inc., Danvers, MA, USA). Signals were visualized using enhanced chemiluminescence western blot detection reagents (GE Healthcare, Little Chalfont, UK) on an ImageQuant LAS 500 imaging device (GE Healthcare). $\beta$-actin was detected using mouse anti- $\beta$-actin monoclonal antibody (mAb; dilution, 1:3,000; Clone AC-74; Sigma-Aldrich Shanghai Trading Co., Ltd., Shanghai, China) and used as an internal control to determine lysate input.

Capacity of rabbit anti-VSTM1 polyclonal antibody $(p A b)$ to cross-link VSTM1-v1. In order to analyze whether the rabbit anti-VSTM1 pAb influenced the functional cross-linking of VSTM1, a reporter construct (pcDB-VSTM1-v1-CD3̧) was generated that expressed a chimeric protein consisting of the extracellular domain of VSTM1-v1 as well as the transmembrane and intracellular domains of $\mathrm{CD} 3 \xi$ through overlapping PCRs as previously described (25). Dual-luciferase reporter assays were then performed. A total of $2 \times 10^{7}$ Jurkat cells were cotransfected with $20 \mu \mathrm{g}$ pcDB-VSTM1-v1-CD3 $\zeta$ or empty vector, $8 \mu \mathrm{g}$ p-nuclear factor kappa-light-chain-enhancer of activated $\mathrm{B}$ cells $(\mathrm{NF} \kappa \mathrm{B})$-Luc plasmid (a construct encoding the firefly luciferase reporter gene under the influence of $\mathrm{NF} \kappa \mathrm{B}$ binding) and $0.4 \mu \mathrm{g}$ p-Renilla luciferase (RL)-thymadine kinase (TK) plasmid (a construct encoding the RL gene; Promega Corporation, Madison, WI, USA) as the internal control. $12 \mathrm{~h}$ post-transfection, cells were stimulated with anti-CD28 (2 $\mu \mathrm{g} / \mathrm{ml}$; clone $15 \mathrm{E} 8)$ in combination with different concentrations $(1,3$ or $5 \mu \mathrm{g} / \mathrm{ml})$ of rabbit anti-VSTM1 pAb or normal rabbit IgG $(5 \mu \mathrm{g} / \mathrm{ml})$ (BD Biosciences, San Jose, CA, USA). Plate-bound anti-CD3 (1 $\mu \mathrm{g} / \mathrm{ml}$; clone OKT3) and tumor necrosis factor- $\alpha(20 \mathrm{ng} / \mathrm{ml}$; eBioscience, San Diego, CA, USA) were used as positive controls. Following $20 \mathrm{~h}$ of stimulation, cells were collected and lysed using Passive Lysis Buffer (Promega Corp.). Firefly and RL activities were measured with the dual-luciferase reporter (DLR) assay system (Promega Corp.) according to the manufacturer's instructions using a Veritas ${ }^{\mathrm{TM}}$ Microplated Luminometer (Promega Corp.).

Cell proliferation assay. Jurkat cells transfected with pCDNA3.1/Myc-His(-)B-VSTM1-v1 (pcDB-VSTM1-v1), or pCDNA3.1/Myc-His(-)B (pcDB) vector plasmids were seeded in 96-well plates at a density of 20,000 per well in the presence or absence of $5 \mu \mathrm{g} / \mathrm{ml}$ anti-VSTM1 pAb or normal rabbit
IgG and then incubated at $37^{\circ} \mathrm{C}$ in a humidified atmosphere with $5 \% \mathrm{CO}_{2}$. Cell proliferation was analyzed using the Cell Counting Kit-8 (CCK-8; Dojindo Molecular Technologies, Inc., Rockville, MA, USA). At 0, 24, 48 and $72 \mathrm{~h}$ post-transfection, $10 \mu \mathrm{l}$ CCK-8 solution was added into each well and incubated for $2 \mathrm{~h}$. Absorbance was measured with a Thermo Scientific Multiskan GO microplate spectrophotometer (Thermo Fisher Scientific Inc., Waltham, MA, USA) at $450 \mathrm{~nm}$ in order to assess the number of viable cells (background absorbance was measured at $630 \mathrm{~nm}$ ). Results were obtained from at least three independent experiments.

Statistical analysis. Values are presented as the mean \pm standard error of the mean/standard deviation. Statistical analysis was performed using the Student's t-test. $\mathrm{P}<0.05$ was considered to indicate a statistically significant difference between values.

\section{Results}

VSTM1 is expressed in peripheral blood leukocytes. A previous study reported that VSTM1 expression was higher in cells of the immune system (7). In the present study qPCR was performed in order to quantify the expression levels of VSTM1. As shown in Fig. 1A, VSTM1 was primarily expressed in immune tissues and cells, including bone marrow and peripheral blood leukocytes. In order to further investigate the expression profile of VSTM1 in leukocytes, RT-qPCR was performed on PBMCs and granulocytes of ten healthy donors. The results showed that VSTM1 was detected in each of the samples (Fig. 1B), and according to the molecular size of the spliced variants, it was found that VSTM1-v1 was the predominantly expressed form. PBLs were then separated from monocytes of the PBMCs in samples from a further three individuals (numbered 1-3) and qPCR was performed in order to quantify the expression levels of VSTM1 in each cell population. As shown in Fig. 1C, VSTM1 was highly expressed in granulocytes of each sample; however, the expression levels of VSTM1 in monocytes and PBL were variable. These results were not consistent with a previous study by Steevels et al (6), in which the monoclonal antibody 1A5 was used to detect VSTM1 expression. This previous study reported that VSTM1 was expressed exclusively by myeloid cells and was absent in lymphoid cells. In order to confirm the existence of VSTM1 in PBLs in the present study, flow cytometric and western blot analyses were performed at the protein level with samples from a further nine donors (numbered 4-12). These results revealed a considerable individual difference in expression levels of VSTM1 among donors. Flow cytometric analysis demonstrated that the proportion of surface VSTM1-v1-positive cells in PBL varied from 4.05 to $50.05 \%$ (Fig. 1D). The representative data from three donors (4, 6 and 11) are shown in Fig. 1E and F. In samples 4 and 11, surface VSTM1-v1 expression was detected with the positive percentages of $50.05 \%$ and $21.72 \%$ respectively; however, in sample 6, VSTM1-v1 was detected in markedly fewer cells $(6.78 \%)$ (Fig. 1E). Consistent results were obtained from representative western blots, which demonstrated a $\sim 60-\mathrm{kDa}$ band of VSTM1 in PBL of donors 4 and 11 , which was, however, not observed in sample 6 . This band was identical to that of the band observed in VSTM1 
A

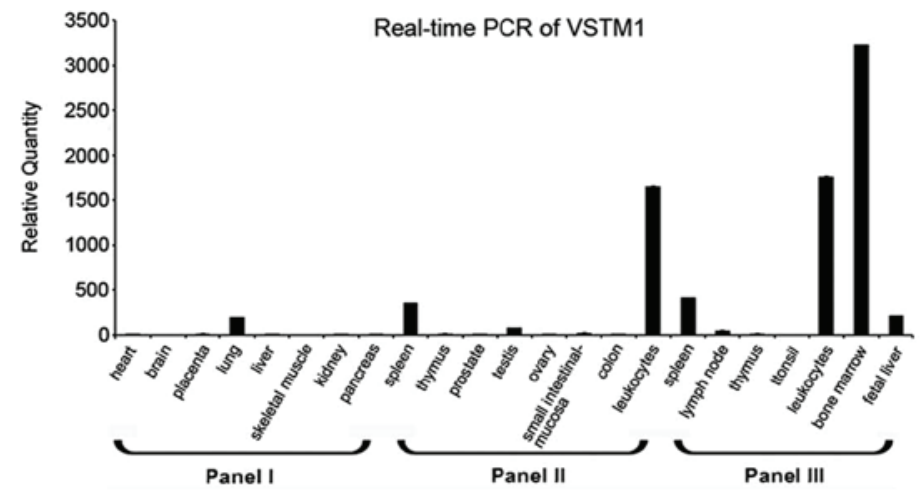

B
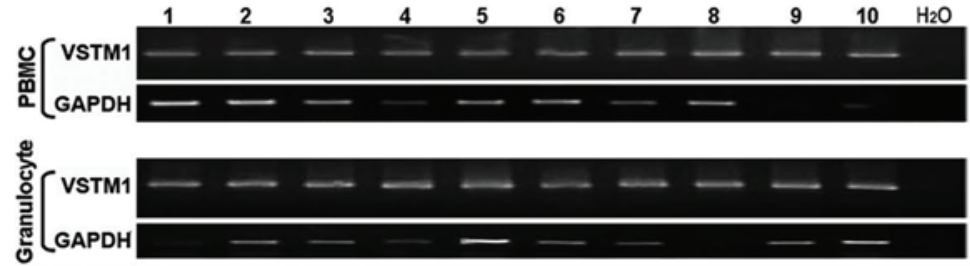

C

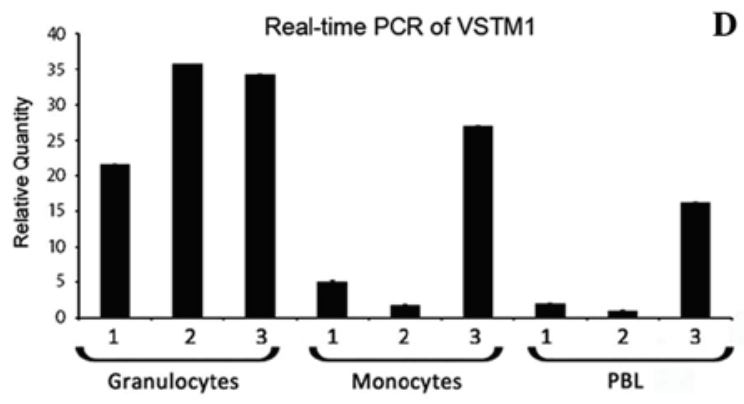

D

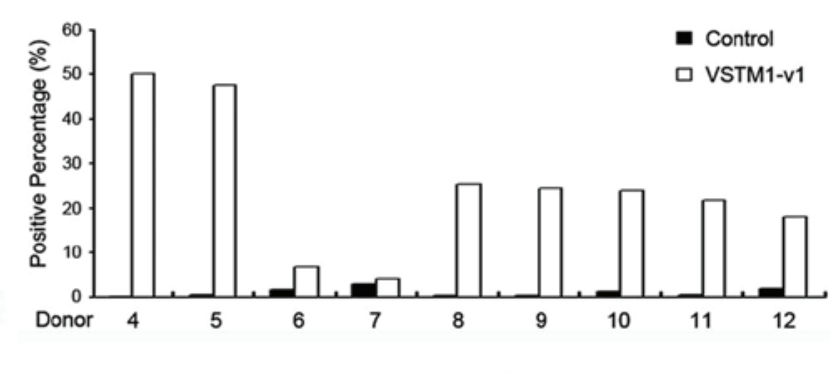

E
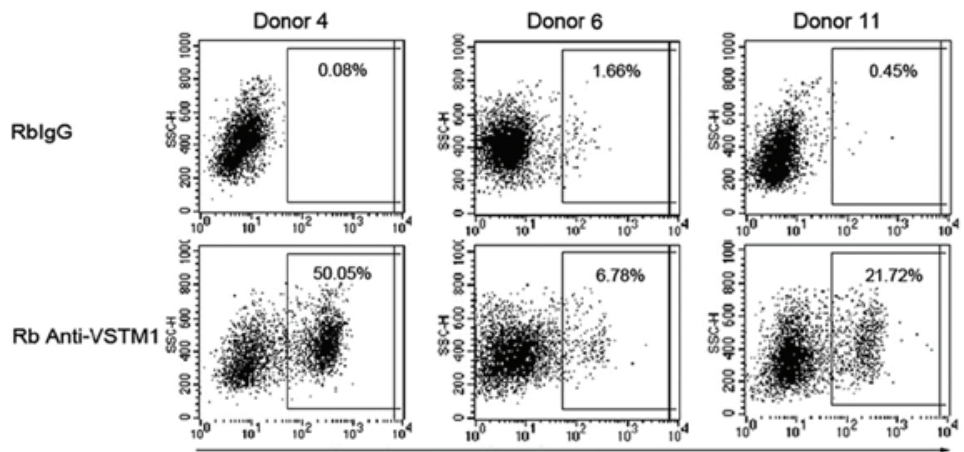

Fluorescence intensity

F

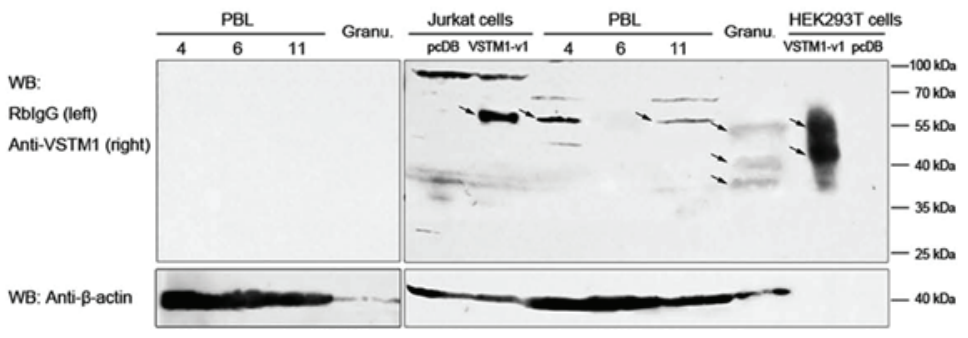

Figure 1. Expression profile of VSTM1. (A) qPCR analysis of VSTM1 expression in numerous tissue type using human MTC ${ }^{\mathrm{TM}}$ panels (I and II; nos. 636742 and 636743, respectively) and human immune system MTC ${ }^{\mathrm{TM}}$ panel (III; no. 636748). Expression levels of VSTM1 were measured relative to levels in the kidneys. (B) RT-qPCR of VSTM1 in peripheral blood mononuclear cells and granulocytes from ten healthy donors. GAPDH was used as the internal control. (C) VSTM1 expression in granulocytes, monocytes and PBLs from three individuals (1-3) was analyzed using qPCR. GAPDH was used as the internal control and the expression levels of VSTM1 were measured relative to those of PBLs from donor two. (D) Flow cytometric analysis of surface VSTM1-v1 expression in PBLs from nine donors (4-12). Quadrants were set relative to RbIgG stainings and positive percentages of VSTM1-v1-expressing cells were counted. (E) Flow cytometric analysis of PBLs of three donors (4, 6 and 11). Images are representative results of the nine independent experiments shown in (D). (F) Western blot analysis of VSTM1-v1 expression in PBLs from donors 4,6 and 11. VSTM1-v1-transfected Jurkat and HEK293T cells and granulocytes were examined as positive controls. Loading volumes of lysates were: PBL, $150 \mu \mathrm{g}$; granulocyte, $150 \mu \mathrm{g}$; Jurkat cells, $100 \mu \mathrm{g}$; and HEK293T, $1 \mu \mathrm{g}$. RbIgG was used as the control antibody and $\beta$-actin was used as the internal control. Arrows indicate specific bands of VSTM1. Values are presented as the mean \pm standard deviation. VSTM1, V-set and transmembrane domain-containing 1; qPCR, quantitative polymerase chain reaction; MTC, mutiple tissue complementary DNA; RT-qPCR, reverse transcription-qPCR; VSTM1-v1, VSTM1 spliced variant 1; PBL, peripheral blood lymphocyte; HEK293T, human embryonic kidney 293T; RbIgG, normal rabbit immunoglobulin G. 


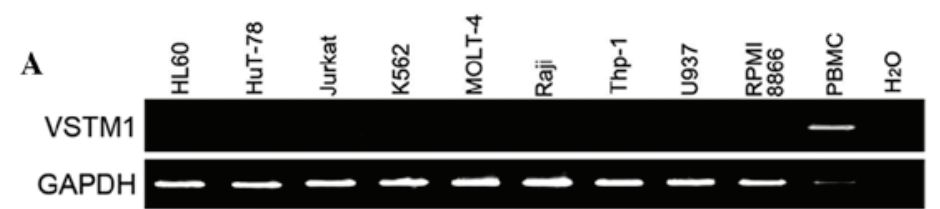

B

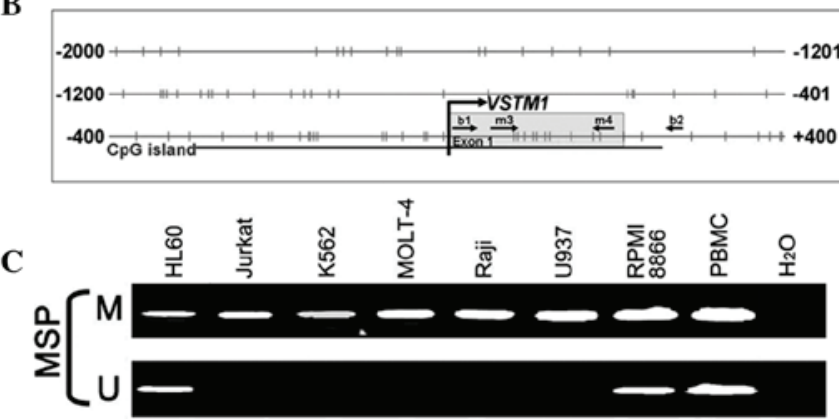

Figure 2. VSTM1 is silenced in numerous hematopoietic tumor cell lines with promoter methylation. (A) RT-PCR of VSTM1 in hematopoietic tumor cell lines. PBMCs were used as a positive control. (B) A CpG island covers the promoter and exon 1 of VSTM1. Horizontal bars, CpG sites; primers for methylation analysis, MSP primers (m3/u3 and m4/u4) and bisulfite genomic sequencing primers (b1 and b2) are indicated; curved arrow, transcription start site. (C) MSP analysis of VSTM1 showed complete methylation of the sites where the MSP primers were located in Jurkat, K562, MOLT4, Raji and U937 cells and partial methylation in HL60 and RPMI 8866 cells, as well as in PBMCs. M, methylated; U, unmethylated; VSTM1, V-set and transmembrane domain-containing 1; RT-PCR, reverse transcription polymerase chain reaction; PBMC, peripheral blood mononuclear cell; MSP, methylation-specific PCR; CpG, cytosine-phosphate-guanine.

overexpressing Jurkat cells; however, the pattern of bands $(\sim 55, \sim 40$ and $\sim 37 \mathrm{kDa})$ in granulocytes was markedly different from that in PBLs. In addition, these results were not comparable to those of a previous study in VSTM1overexpressing HEK293T cells, which reported bands at $\sim 55$ and $\sim 45 \mathrm{kDa}$ (21). This therefore indicated that VSTM1 was diversely modified in cells of different origin.

VSTM1 is silenced following promoter methylation in numerous hematopoietic tumor cell lines. VSTM1 was found to be broadly expressed in normal human PBLs; therefore, RT-qPCR was performed in order to further investigate whether VSTM1 was also expressed in hematopoietic tumor cell lines. The results showed that VSTM1 was silenced in the majority of the examined cell lines (Fig. 2A). The aberrant promoter $\mathrm{CpG}$ methylation was previously reported to be associated with gene silencing (16); therefore, in the present study, the VSTM1 promoter was screened for $\mathrm{CpG}$ islands using the CpG Island Searcher. The results indicated that the VSTMI promoter contained a typical $\mathrm{CpG}$ island (Fig. 2B). VSTMI promoter methylation was then analyzed using MSP and it was demonstrated that the promoters of VSTMI-silenced cell lines were methylated to varying degrees (Fig. 2C). This therefore indicated that there was an association between the loss of VSTM1 and CpG hypermethylation in the promoter region.

Expression of VSTM1 was restored following pharmacological demethylation. Promoter hypermethylation was performed in Jurkat cells in order to silence VSTM1; cells were then treated with the methyltransferase inhibitor 5-aza-2'-deoxycytidine alone or combination with trichostatin A, a histone deacetylase inhibitor. This treatment was shown to restore mRNA and protein expression levels of VSTM1 (Fig. 3A and B); in addition, MSP analysis demonstrated that this treatment resulted in promoter demethylation (Fig. 3C) and BGS revealed specific demethylation of individual $\mathrm{CpG}$ sites (Fig. 3D). These results indicated that VSTM1 silencing in Jurkat cells was due to epigenetic regulation.

Overexpression of VSTM1-vl inhibits Jurkat cell growth. Results of the present study showed that VSTM1 was expressed in normal human leukocytes and silenced in hematopoietic tumor cells; therefore, subsequent experiments were performed in order to determine effects of exogenous VSTM1-v1 expression on the growth of Jurkat cells following VSTM1 promoter hypermethylation and gene silencing. As shown in Fig. 1E, VSTM1-v1 was successfully expressed in Jurkat cells following transfection. CCK-8 assays revealed that VSTM1-v1 inhibited the growth of Jurkat cells compared with that of vector-transfected control cells (Fig. 4A). VSTM1-v1 is a type I transmembrane protein, which contains two cytoplasmic ITIMs. Therefore, it was hypothesized that ligand interaction with the extracellular region may induce the transmission of an inhibitory signal into the cell; however, ligands for VSTM1-v1 have not yet been identified. Therefore, in the present study, the VSTM1 antibody was used as an agonist to bind to the extracellular region of VSTM1. The influence of rabbit anti-VSTM1 pAb on the functional cross-linking of VSTM1 was determined using NFkB-firefly luciferase reporter assays in Jurkat cells with the construct expressing the VSTM1-v1-CD3ל chimera (25). Incubation with the anti-VSTM1 pAb instead of normal rabbit IgG and in the presence of anti-CD28 resulted in NFאB activation and consequently increased firefly luciferase activity. In addition, firefly luciferase activity was enhanced following increased doses of the anti-VSTM1 pAb (Fig. 4B), which indicated that this antibody was capable of cross-linking VSTM1-v1. VSTM1-v1- or vector-transfected Jurkat cells were then treated with the anti-VSTM1 pAb or normal rabbit IgG (Fig. 4C). A CCK8 assay showed that incubation with the anti-VSTM1 $\mathrm{pAb}$ further augmented the inhibitory effect of VSTM1-v1 on Jurkat cell growth. 
A

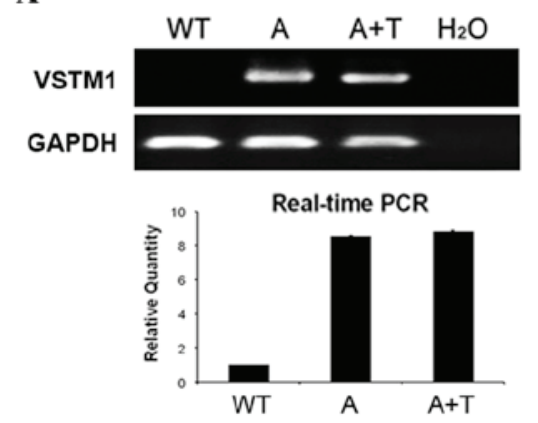

C

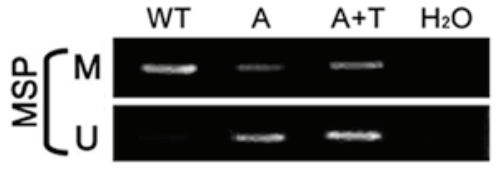

B
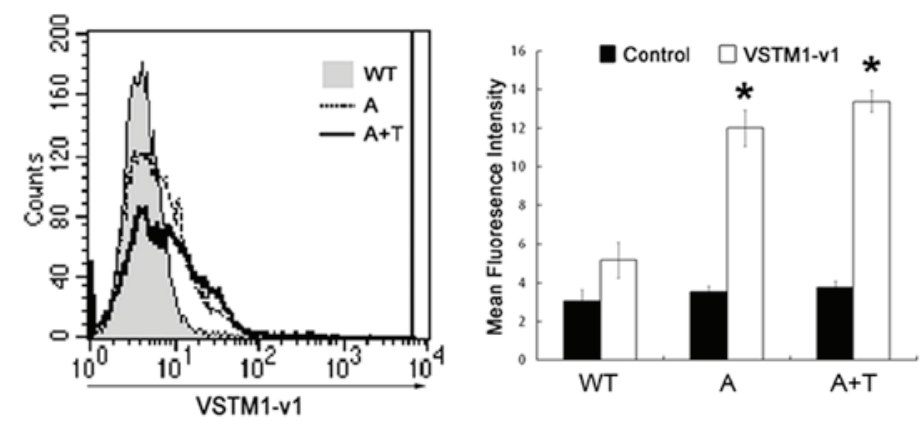

D
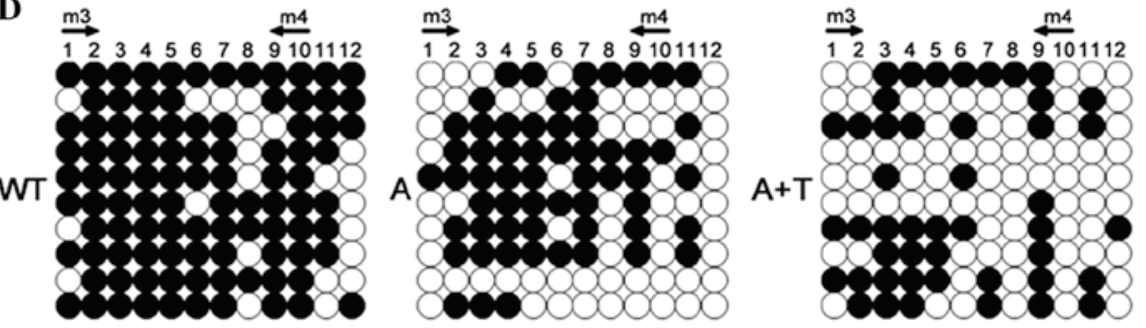

Figure 3. Pharmacological demethylation restores VSTM1 expression in Jurkat cells. (A) Semiquantitative RT-PCR and qPCR analyses using WT Jurkat cells and Jurkat cells treated with the methyltransferase inhibitor A alone or in combination with the histone deacetylase inhibitor T. GAPDH was used as an internal control. (B) Flow cytometric analysis confirmed the restoration of surface VSTM1-v1 expression in Jurkat cells following pharmacological demethylation. Representative results and the mean fluorescence intensity of VSTM1 expression for three independent experiments. Normal rabbit immunoglobulin G was used for control staining. Values are presented as the mean \pm standard error of the mean. "P $<0.05$ vs. WT cells. (C) MSP verified the pharmacological demethylation of the VSTM1 promoter. (D) Detailed bisulfite genomic sequencing analysis of the VSTM1 promoter. Circles, CpG sites analyzed; row of circles, an individual promoter allele that was cloned, randomly selected and sequenced; filled circle, methylated $\mathrm{CpG}$ site; open circle, unmethylated $\mathrm{CpG}$ site. A, 5-aza-2'-deoxycytidine; T, trichostatin A; WT, wild-type; VSTM1, V-set and transmembrane domain-containing 1; RT-PCR, reverse transcription polymerase chain reaction; qPCR, quantitative PCR; VSTM1-v1, VSTM1 spliced variant 1; MSP, methylation-specific PCR; CpG, cytosine-phosphate-guanine.

A

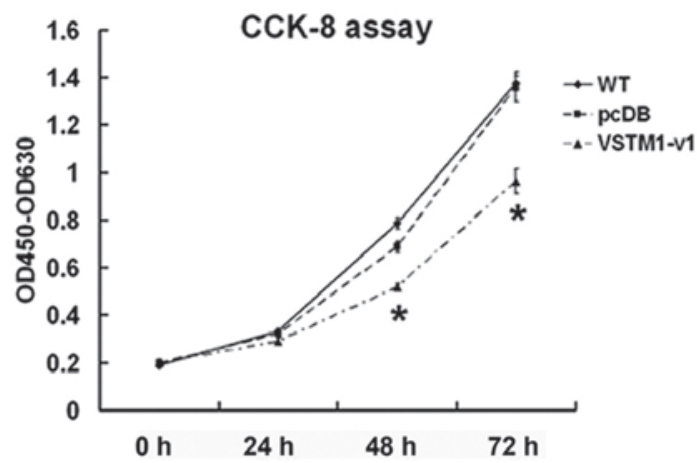

B

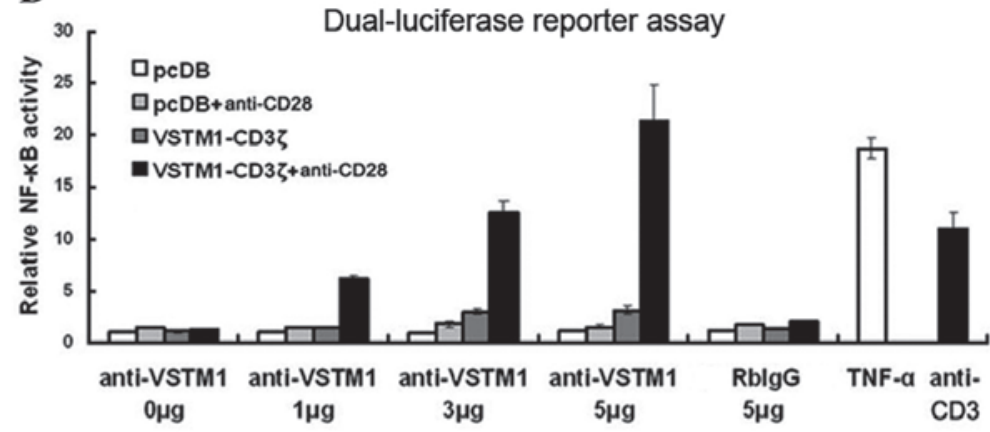

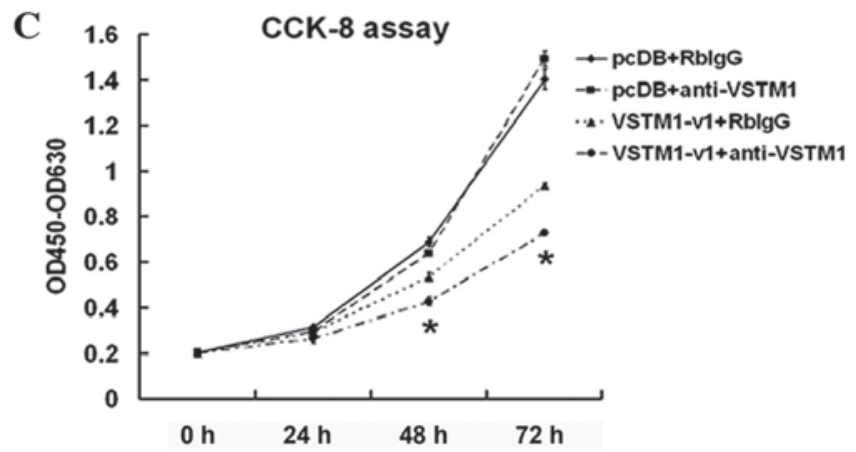

Figure 4. Overexpression of VSTM1-v1 inhibits Jurkat cell growth. (A) Representative results of $\geq$ five independent CCK-8 assays in WT, pcDB- and VSTM1-v1-transfected cells. Values are presented as the difference between absorbance at 450 and $630 \mathrm{~nm}$ (OD450-OD630). (B) NFkB-firefly luciferase reporter assays in Jurkat cells demonstrated the capacity of rabbit anti-VSTM1 pAb to cross-link VSTM1-v1. Representative results from three individual experiments are shown. Relative NFкB activity was the mean of the ratio of firefly to renilla luciferase activities \pm SD. TNF- $\alpha$ and plate-bound anti-CD3 were used as positive controls for activating NFkB. (C) CCK-8 assays of VSTM1-v1- and pcDB-transfected Jurkat cells following interaction with anti-VSTM1 pAb. RbIgG control was used as the internal control. Anti-VSTM1 pAb was shown to further inhibit the growth of VSTM1-v1-transfected cells. Representative result of three independent experiments. Values are presented as the mean $\pm \mathrm{SD}$. "P $<0.05$ vs. controls at each time-point. VSTM1, V-set and transmembrane domain-containing 1; VSTM1-v1, VSTM1 spliced variant 1; CCK-8, cell counting kit 8; WT, wild-type; NFkB, nuclear factor kappa-lightchain-enhancer of activated B cells; pAb, polyclonal antibody; TNF- $\alpha$, tumor necrosis factor- $\alpha$; pcDB, pcDB-VSTM1-v1-CD3 $\zeta$ reporter construct; RbIgG, normal rabbit immunoglobulin G; SD, standard deviation. 


\section{Discussion}

VSTMI is a potential leukocyte differentiation antigen gene that was previously identified using immunogenomics. VSTMI encodes numerous splicing isoforms, of which VSTM1-v1 is the most predominant and widely expressed form (7). VSTM1-v1 is a type I transmembrane molecule that contains an extracellular IgV-like domain and two ITIMs in its cytoplasmic region. VSTM1 was previously reported to be expressed exclusively in myeloid cells and absent in lymphoid cells as indicated by detection using the monoclonal antibody 1A5 (6). However, in the present study, VSTM1 mRNA expression was observed not only in granulocytes and monocytes but also in PBLs from several healthy donors using RT-qPCR; in addition, there were considerable differences in VSTMI expression levels among individuals. Flow cytometric analysis using rabbit anti-VSTM1 $\mathrm{pAb}$ confirmed the expression patterns at the protein level. However, the results of the western blot analysis may provide a possible explanation for the contradictory results from the present and previous study about the expression of VSTM1 in PBLs. The present study determined that the molecular size of VSTM1 detected in PBLs was consistent with that of the overexpressed VSTM1-v1 in Jurkat cells, which was different from that of endogenous VSTM1 in granulocytes and overexpressed VSTM1-v1 in HEK293T cells (21). This indicated that VSTM1 may be diversely modified in cells of different origins, with different molecular sizes and conformations; therefore, the application of monoclonal antibodies for the detection of this molecule is likely to be limited as a monoclonal antibody only recognizes a single epitope of a molecule (26).

VSTM1 is broadly expressed in normal human PBLs; however, it was found to be silenced in multiple leukemia cell lines. VSTM1 is located on chromosome 19q13.4, a genomic region widely reported to be prone to genetic and epigenetic modifications in numerous hematopoietic malignancies (8-12). In the present study, a bioinformatic search tool was used, which indicated that a typical $\mathrm{CpG}$ island was present in the promoter of VSTM1. RT-PCR and MSP analyses revealed that there was a link between the expression of silenced VSTM1 in hematopoietic tumor cell lines and $\mathrm{CpG}$ hypermethylation in the promoter region. This therefore confirmed that epigenetic modifications were an important mechanism in the regulation of VSTM1, which may be of use as a promising novel diagnostic and prognostic genetic marker for hematopoietic malignancies (27). However, the involvement of genetic alterations in this process remains to be elucidated.

Previous studies have identified numerous inhibitory receptors containing ITIMs, which prevent excessive immune reactions and autoimmunity, thereby maintaining the balance of the immune system (28-30). These studies may therefore indicate the function of the two ITIMs in the cytoplasmic tail of VSTM1-v1. Following interaction with the extracellular portion of the receptor by ligands, cytoplasmic ITIMs bind to the sarcoma homology 2 domain of phosphatases (SHP), resulting in the inactivation of different kinases and downregulation of cell activation (28-30). ITIMs in VSTM1-v1 were also reported to recruit SHP-1 and SHP-2, which inhibited the fragment, crystallizable epsilon receptor I-mediated signaling involved in reactive oxygen species production and the microbicidal activity of phagocytes $(6,31)$. Further studies are required to determine whether the inhibitory effect of VSTM1-v1 on cell growth may also be achieved via SHP recruitment through its cytoplasmic ITIMs.

In conclusion, the results of the present study demonstrated that VSTM1 was expressed in myeloid cells as well as lymphocytes, which were of different molecular size and exhibited considerable variation in their expression levels among donors. VSTM1 was found to be silenced in numerous hematopoietic malignancy cell lines, with $\mathrm{CpG}$ hypermethylation within the promoter; however, pharmacological demethylation in Jurkat cells was shown to be able to restore VSTM1 expression. In addition, overexpression of VSTM1-v1 in Jurkat cells inhibited cell growth, which was further restricted following the interaction with the extracellular portion of VSTM1-v1 using anti-VSTM1 antibodies. These results therefore elucidated the expression regulation and functional roles of VSTMI in the inhibition of hematopoietic tumor cells, which may contribute towards the development of novel diagnostic and treatment strategies for hematopoietic malignancies.

\section{Acknowledgements}

The present study was supported by grants from the Specialized Research Fund for the Doctoral Program of Higher Education of China (no. 20110001110016) and the National Natural Science Foundation of China (no. 31400736).

\section{References}

1. Zola H: Human leukocyte differentiation antigens as therapeutic targets: the CD molecules and CD antibodies. Expert Opin Biol Ther 1: 375-383, 2001 .

2. Zola $H$ and Swart B: The human leucocyte differentiation antigens (HLDA) workshops: the evolving role of antibodies in research, diagnosis and therapy. Cell Res 15: 691-694, 2005.

3. Bisig B, Gaulard P and de Leval L: New biomarkers in T-cell lymphomas. Best Pract Res Clin Haematol 25: 13-28, 2012.

4. Huang PY, Best OG, Almazi JG, et al: Cell surface phenotype profiles distinguish stable and progressive chronic lymphocytic leukaemia. Leuk Lymphoma 55: 2085-2092, 2014.

5. Walter RB, Appelbaum FR, Estey EH and Bernstein ID: Acute myeloid leukemia stem cells and CD33-targeted immunotherapy. Blood 119: 6198-6208, 2012.

6. Steevels TA, Lebbink RJ, Westerlaken GH, Coffer PJ and Meyaard L: Signal inhibitory receptor on leukocytes-1 is a novel functional inhibitory immune receptor expressed on human phagocytes. J Immunol 184: 4741-4748, 2010.

7. Guo X, Zhang Y, Wang P, et al: VSTM1-v2, a novel soluble glycoprotein, promotes the differentiation and activation of Th17 cells. Cell Immunol 278: 136-142, 2012.

8. Brambillasca F, Mosna G, Colombo M, et al: Identification of a novel molecular partner of the E2A gene in childhood leukemia. Leukemia 13: 369-375, 1999.

9. Di Bernardo MC, Crowther-Swanepoel D, Broderick P, et al: A genome-wide association study identifies six susceptibility loci for chronic lymphocytic leukemia. Nat Genet 40: 1204-1210, 2008.

10. Chaudhary K, Deb S, Moniaux N, Ponnusamy MP and Batra SK: Human RNA polymerase II-associated factor complex: dysregulation in cancer. Oncogene 26: 7499-7507, 2007.

11. Fuchs O, Provaznikova D, Kocova M, et al: CEBPA polymorphisms and mutations in patients with acute myeloid leukemia, myelodysplastic syndrome, multiple myeloma and non-Hodgkin's lymphoma. Blood Cells Mol Dis 40: 401-405, 2008.

12. Abdool A, Donahue AC, Wohlgemuth JG and Yeh CH: Detection, analysis and clinical validation of chromosomal aberrations by multiplex ligation-dependent probe amplification in chronic leukemia. PLoS One 5: e15407, 2010.

13. Kunitz A, Wolter M, van den Boom J, et al: DNA hypermethylation and aberrant expression of the EMP3 gene at 19q13.3 in human gliomas. Brain Pathol 17: 363-370, 2007. 
14. Zhang Y, Bhat I, Zeng M, et al: Human kallikrein 10, a predictive marker for breast cancer. Biol Chem 387: 715-721, 2006.

15. Roman-Gomez J, Jimenez-Velasco A, Agirre X, et al: The normal epithelial cell-specific 1 (NES1) gene, a candidate tumor suppressor gene on chromosome 19q13.3-4, is downregulated by hypermethylation in acute lymphoblastic leukemia. Leukemia 18: 362-365, 2004.

16. Rüter B, Wijermans PW and Lübbert M: DNA methylation as a therapeutic target in hematologic disorders: recent results in older patients with myelodysplasia and acute myeloid leukemia. Int J Hematol 80: 128-135, 2004.

17. Claus R and Lübbert M: Epigenetic targets in hematopoietic malignancies. Oncogene 22: 6489-6496, 2003.

18. Zhang M, Xiao XQ, Jiang YF, et al: DNA demethylation in PD-1 gene promoter induced by 5 -azacytidine activates PD-1 expression on Molt-4 cells. Cell Immunol 271: 450-454, 2011.

19. Gattazzo C, Teramo A, Miorin M, et al: Lack of expression of inhibitory KIR3DL1 receptor in patients with natural killer cell-type lymphoproliferative disease of granular lymphocytes. Haematologica 95: 1722-1729, 2010

20. Li T, Zhong J, Chen Y, et al: Expression of chemokine-like factor 1 is upregulated during $\mathrm{T}$ lymphocyte activation. Life Sci 79: 519-524, 2006

21. Li T, Wang W, Chen Y and Han W: Preparation and characterization of monoclonal antibodies against VSTM1. Monoclon Antib Immunodiagn Immunother 32: 283-289, 2013.

22. Shao L, Cui Y, Li H, et al: CMTM5 exhibits tumor suppressor activities and is frequently silenced by methylation in carcinoma cell lines. Clin Cancer Res 13: 5756-5762, 2007.
23. Wang Y, Li J, Cui Y, et al: CMTM3, located at the critical tumor suppressor locus 16q22.1, is silenced by CpG methylation in carcinomas and inhibits tumor cell growth through inducing apoptosis. Cancer Res 69: 5194-5201, 2009.

24. Li T, Guo XH, Wang PZ, Song QS, Ma DL and Han WL: Preparation, purification, and characterization of the polyclonal antibody against human VSTM1. Xi Bao Yu Fen Zi Mian Yi Xue Za Zhi 28: 1291-1294, 2012 (In Chinese).

25. Lebbink RJ, de Ruiter T, Adelmeijer J, et al: Collagens are functional, high affinity ligands for the inhibitory immune receptor LAIR-1. J Exp Med 203: 1419-1425, 2006.

26. Kammerer R1, Hahn S, Singer BB, Luo JS and von Kleist S: Biliary glycoprotein (CD66a), a cell adhesion molecule of the immunoglobulin superfamily, on human lymphocytes: structure, expression and involvement in T cell activation. Eur J Immunol 28: 3664-3674, 1998.

27. Ushijima T: Detection and interpretation of altered methylation patterns in cancer cells. Nat Rev Cancer 5: 223-231, 2005.

28. Ravetch JV and Lanier LL: Immune inhibitory receptors. Science 290: 84-89, 2000.

29. Bléry M, Olcese L and Vivier E: Early signaling via inhibitory and activating NK receptors. Hum Immunol 61: 51-64, 2000.

30. Daëron M, Jaeger S, Du Pasquier L and Vivier E: Immunoreceptor tyrosine-based inhibition motifs: a quest in the past and future. Immunol Rev 224: 11-43, 2008.

31. Steevels TA, van Avondt K, Westerlaken GH, et al: Signal inhibitory receptor on leukocytes-1 (SIRL-1) negatively regulates the oxidative burst in human phagocytes. Eur J Immunol 43: 1297-1308, 2013. 